This item was submitted to Loughborough's Research Repository by the author.

Items in Figshare are protected by copyright, with all rights reserved, unless otherwise indicated.

\title{
Disturbance observer based control for gust alleviation of a small fixed-wing
} UAS

PLEASE CITE THE PUBLISHED VERSION

http://dx.doi.org/10.1109/ICUAS.2016.7502575

PUBLISHER

(C) IEEE

VERSION

AM (Accepted Manuscript)

LICENCE

CC BY-NC-ND 4.0

REPOSITORY RECORD

Smith, Jean, Cunjia Liu, and Wen-Hua Chen. 2016. "Disturbance Observer Based Control for Gust Alleviation of a Small Fixed-wing UAS". figshare. https://hdl.handle.net/2134/22715. 


\title{
Disturbance Observer Based Control for Gust Alleviation of a Small Fixed-Wing UAS
}

\author{
Jean Smith, Cunjia Liu and Wen-Hua Chen \\ Department of Automotive and Aeronautical Engineering \\ Loughborough University, Loughborough, LE11 3TQ, UK \\ Email: J.Smith5@1boro.ac.uk, C.Liu5@lboro.ac.uk,W.chen@lboro.ac.uk
}

\begin{abstract}
This paper outlines a method of applying a linear disturbance observer to a small Unmanned Aerial System (UAS) to reduce the influence of unpredictable gusts on the non-linear aircraft dynamics. This work aims to show that by using a linear state-space model it is possible to estimate external disturbances and use the available control surfaces to alleviate the influence of gusts on aircraft dynamics accordingly. This paper focuses on the longitudinal channel of a small UAS to demonstrate the strategy. A baseline Linear Quadratic Regulator with Integral Action (LQI) is first developed; the disturbance observer based control strategy is then patched into this baseline controller to demonstrate the performance improvement. Simulations are conducted using the designed linear observer to alleviate various disturbance sources on a fully non-linear simulation of the UAS. This aims to demonstrate performance of an observer in a realistic situation where uncertainties between the linear observer and non-linear plant to be controlled are present.
\end{abstract}

\section{INTRODUCTION}

The use of small UASs is an area of significant interest and is expanding in both scale and range of applications. For these systems, linear control schemes are still very common as, especially for fixed wing aircraft, a reasonable linear model can be obtained quite easily. A common problem for these UASs being deployed outdoors is the effect of wind and gusting. This is far more significant for small UASs when compared to large general aviation aircraft for multiple reasons. Firstly, the reduced size and associated inertia mean the same gust magnitudes result in greater disturbances [1]. Secondly, the airspeed at which these UASs operate are of the same order of magnitude as the wind and gust velocity [2], greatly increasing the effect on the dynamics. Although research has been undertaken into specific sensors for sensing oncoming disturbances [3] [4], the systems require additional sensors beyond what is commonly found on small UASs. This generally results in more complex systems and additional expense, which can be very significant depending on the additional sensor(s) employed. The ideal solution is a system which does not require additional sensors or modification to the aircraft itself to function. This paper aims to demonstrate the feasibility of using Disturbance Observer Based Control (DOBC) to solve this problem. DOBC uses a model of the system to predict changes in state based on current state and control input; this prediction is then compared to the measured change in state, using the difference in response to estimate disturbances. Designing an appropriate control scheme to act on these disturbance estimates allows for feedforward disturbance rejection. DOBC has the significant benefit of allowing 'patching' into a baseline controller. This means the baseline controller does not need to be designed specifically to work with a Disturbance Observer (DO), and even allows retrofitting of the technique to existing controllers. With a well designed DO, the baseline controller performance is not affected when no disturbance is present and is significantly improved when disturbances are present. With a model which perfectly represents the physical system, the DO prediction would match exactly with the system state if there is no external disturbance on the system. Alternatively, any change in state which an ideal DO did not predict could therefore be attributed to an external disturbance, hence the name of the technique. Using these disturbance estimates, a control strategy can be designed which aims to mitigate the effect of the disturbance on the system. Take for example an aircraft in straight and level flight. If a sudden increase in airspeed and pitch rate is measured with no change in control input, the DO could attribute this to a gust; the complimenting control strategy would generate a compensation signal to alleviate the perturbation on the aircraft that such a gust would normally generate more quickly than traditional feedback control. Therein lies the overall premise of the presented method.

Such performance does rely on a very accurate model which in reality can prohibitively difficult or expensive to obtain, especially in the context of small UASs operated by individuals or small companies. However, this work aims to show that using a simple linear model (which is more easily obtained) with inaccuracies can still be used to significantly improve gust alleviation. The proposed method uses a linear DO to rapidly estimate an external disturbance (gust) and generate a compensation signal to mitigate the physical response. This will be of great benefit to a wide range of UASs as their use as a sensing platform is extremely prevalent; having a more stable platform could significantly improve the quality of the collected data.

Furthermore, good rejection of position disturbances (such as height used in this work) provides the facility to improve path following for small UASs in turbulent, gusting weather conditions. This is an area of interest for future work. Certain 


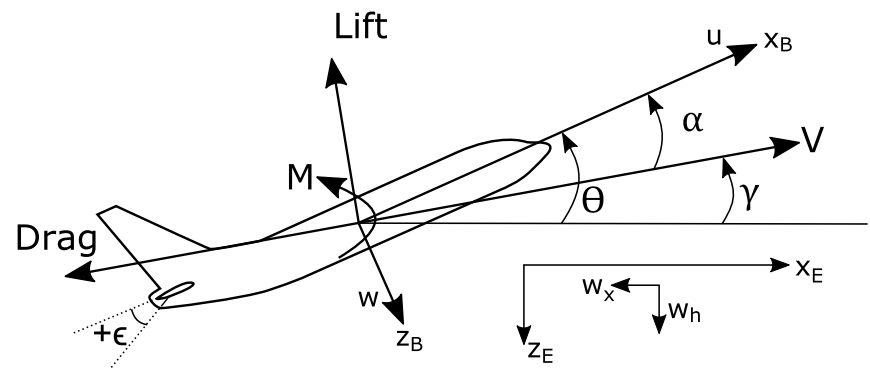

Fig. 1: The coordinate system and control direction employed in the longitudinal controller design process implemented in this paper.

scenarios, such as landing, are very critical on airspeed and position. A system which is able to minimise the effect of gusts on these parameters could significantly improve flight safety. The aim of using a DO is to maximise the range of platforms which can benefit from the technique by not requiring additional hardware. Furthermore, as the control calculations do not become significantly more complex with the employed linear DO, it may be possible to embed the control scheme into existing commercially available UAS flight controllers such as the open source code used on the ArduPilot Mega (APM) or Pixhawk, to allow for low latency operation in flight testing.

\section{Aircraft Modelling}

\section{A. Coordinate System Definition}

To properly demonstrate controller design, an appropriate coordinate system is first required. An important part of this system is proper definition of the positive wind directions. Although wind does not act in distinct axes, it is helpful to break it down into individual components to better understand the impact on the aircraft. Fig 1 details the chosen coordinate system, where $x_{B}, z_{B}$ are the body axes, $x_{E}, z_{E}$ are the Earth reference horizontal and vertical axes, $w_{x}, w_{h}$ are positive horizontal and vertical wind directions, $u, w$ and $V$ are body axes forward and vertical velocity and airspeed, $M$ is the pitching moment, $\alpha$ is aircraft angle of attack, $\theta$ is aircraft pitch angle and $\gamma$ is the flight path angle while Lift and Drag use their standard meanings.

\section{B. Aircraft Coefficients}

The aircraft used in this study is a Skywalker X8, as shown in Fig 2. This aircraft is fitted with an ArduPilot Mega (APM) flight controller. The APM employs a 6 axis IMU providing body axis acceleration and angular rate measurements, as well as an external magnetometer, GPS and pitot tube for measuring airspeed. The $\mathrm{X} 8$ is only equipped with elevons and an engine; no direct yaw control is available. Further detail on this system is given in [5].

To obtain a good model of the Skywalker X8 used, the aircraft geometry was modelled in SimGen ${ }^{1}$, which combines Vortex Lattice calculations with empirical aircraft data to

\footnotetext{
${ }^{1}$ http://www.bihrle.com/simgen/index.dna
}

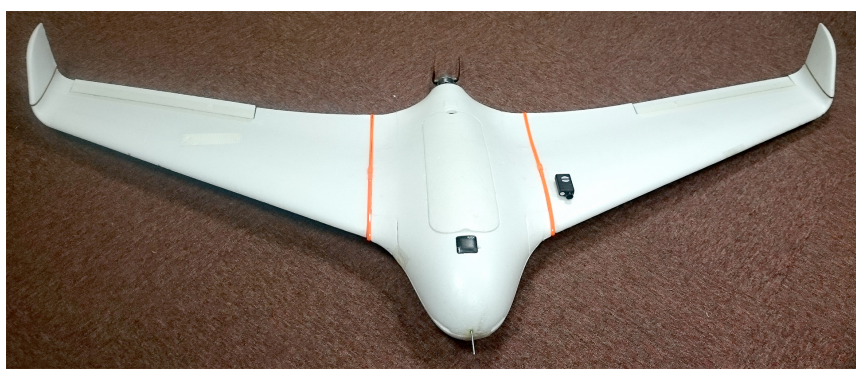

Fig. 2: The Skywalker $\mathrm{X} 8$ used in the modelling process. Wingspan: $2.12 \mathrm{~m}$, Weight $2.95 \mathrm{~kg}$.

return data tables of aircraft coefficients at various flight conditions. The output from SimGen comes in the form of large 2D discrete data tables for each of the aircrafts' coefficients. The coefficients are given for combinations of angle of attack and sideslip. Although this data is well suited to a flight simulation environment, the discrete nature does not lend itself to use in control system design. To account for this, the next step in the process was to transfer these tables into linearised equations. This was done through analytical analysis of the numbers themselves and theoretical knowledge of the significance of the coefficient to determine how much simplification could be applied. Eq (1) gives an example output of this process in coefficient form, where $X$ represents the force in the body $\mathrm{x}$ axis direction, $f(X)$ represents the linear coefficient denoted by the subscript, $\alpha$ is angle of attack, $\beta$ is angle of sideslip, $\epsilon$ is control surface deflection, $q$ is pitch rate, $\bar{q}$ is dynamic pressure and $S$ is the lifting surface reference area. Similar equations were created for each force and moment source.

$$
\begin{gathered}
X=C_{x} \bar{q} S= \\
{\left[f\left(X_{\alpha}\right) \cdot \alpha+f\left(X_{\beta}\right) \cdot \beta+f\left(X_{\epsilon}\right) \cdot \epsilon+f\left(X_{q}\right) \cdot q\right] \bar{q} S}
\end{gathered}
$$

\section{State-Space Model}

A linear state-space model of the aircraft was chosen to be used in control design. State-space modelling is widely used where a linear model is required and the matrix format allows for efficient computation. The model follows the standard form shown in (2) where $A$ is the state matrix, $B$ is the control matrix, $C$ is the output matrix and $D$ is the feed through matrix (which is omitted in this work). The state matrices are detailed in (3). This is based on models used in [6].

$$
\begin{aligned}
& \dot{x}=A x+B u \\
& y=C x+D u
\end{aligned}
$$




$$
\begin{aligned}
& A=\left[\begin{array}{ccccc}
X_{u} & X_{w} & X_{q} & -g \cos \theta^{*} & 0 \\
Z_{u} & Z_{w} & Z_{q} & -g \sin \theta^{*} & 0 \\
M_{u} & M_{w} & M_{q} & 0 & 0 \\
0 & 0 & 1 & 0 & 0 \\
\sin \theta^{*} & -\cos \theta^{*} & 0 & u^{*} \cos \theta^{*}+ & 0 \\
& & & w^{*} \sin \theta^{*} &
\end{array}\right] \\
& x=\left[\begin{array}{lllll}
u & w & q & \theta & h
\end{array}\right]^{T} \\
& B=\left[\begin{array}{cc}
0 & X_{\delta t} \\
Z_{\delta \epsilon} & 0 \\
M_{\delta \epsilon} & 0 \\
0 & 0 \\
0 & 0
\end{array}\right] \\
& u=\left[\begin{array}{ll}
\delta_{\epsilon} & \delta_{t}
\end{array}\right]^{T} \\
& C=\left[\begin{array}{lllll}
1 & 0 & 0 & 0 & 0 \\
0 & 0 & 0 & 0 & 1
\end{array}\right]
\end{aligned}
$$

Where $q$ is the pitch rate, $h=-z_{E}$ the height above ground, $X, Z, M$ are the force and moment coefficients due to their respective subscripts, $g$ is gravity and $\delta_{\epsilon}, \delta_{t}$ are the elevator and throttle control inputs. Any variable with an attached asterisk denotes the state of that variable at the trim condition about which the state-space model was created. For this paper, this was chosen as straight and level flight at $V=15 \mathrm{~m} / \mathrm{s}$, which represents a common cruise condition for the Skywalker X8. It is pertinent to note that the $X_{\delta \epsilon}$ which is usually a part of the system has been omitted from the $B$ matrix. This term is relatively small in comparison to the included terms. Furthermore, removing it provides an additional inaccuracy in the system. This should further demonstrate the applicability and robustness of the technique with uncertainties present.

The outputs to be regulated by the controller are forward speed and height, as shown by the $C$ matrix.

To obtain the trim condition, (4) and (5) are solved to determine the equilibrium condition for the specified cruise.

$$
\begin{gathered}
\delta_{\epsilon}^{*}=\frac{-C_{M 0}-C_{M_{\alpha}} \alpha^{*}}{C_{M \delta_{\epsilon}}} \\
\delta_{T}^{*}=\frac{2 m(g \sin \theta)-\rho\left(V_{a}^{*}\right)^{2} S\left[C_{X} \alpha^{*}+C_{X_{\delta \epsilon}} \alpha^{*} \delta_{\epsilon}^{*}\right]}{\rho S_{\text {prop }} C_{\text {prop }} k_{\text {motor }}^{2}}+ \\
\frac{\left(V_{a}^{*}\right)^{2}}{k_{\text {motor }}^{2}}
\end{gathered}
$$

Where $C_{M}$ represents the pitching moment due to the associated subscript.

The states chosen for the model are commonly used in state-space aircraft modelling, with the exception of $h$. The inclusion of a kinematic height calculation allows the disturbance observer to counter vertical disturbances introduced by gusts. This showcases the potential for DOs to be used for improved path following in gusting conditions as well as attitude stabilisation. An accurate measure of height is commonly available for small UASs as most are equipped with an on-board barometer.
The sensors commonly found on small UASs do not allow for direct measurement of $u$ and $w$ body velocities; it is more common to measure total airspeed by means of a pitot probe. However, methods exist in literature [7] which allow for the estimation of $\alpha$ and $\beta$ angles using conventional sensors aboard small UASs. With this information, $u$ and $w$ can be extrapolated from $V_{a}$, which is commonly measured on small UASs. This, or a similar method, would need to be implemented on the test platform to translate the control scheme presented herein to flight tests.

\section{Simulation EnVironment}

The simulation environment employed uses the built in Simulink 6 degree-of-freedom (6DOF) simulation model as the core of the environment. This system is part of the Aerospace modelling toolbox and provides a set of mathematical calculations to simulate aircraft flight based on aircraft coefficients. Examples are supplied by Mathworks ${ }^{2}$ and similar environments can be found in a wide range of literature concerning flight simulation [8] [9] [10] [11]. A brief overview of the key features is given in this section.

Eq. (1) and the remaining forces and moments acting on the aircraft are collected as shown in (6) and (7), where $b$ is the wingspan of the aircraft and $\bar{c}$ is the mean aerodynamic chord. To translate the forces into motion, the equations are written as shown in (8) and (9).

$$
\begin{gathered}
{\left[\begin{array}{l}
X \\
Y \\
Z
\end{array}\right]=\left[\begin{array}{l}
C_{x} \\
C_{y} \\
C_{z}
\end{array}\right] \bar{q} S} \\
{\left[\begin{array}{c}
L \\
M \\
N
\end{array}\right]=\left[\begin{array}{c}
C_{L} \\
C_{M} \\
C_{N}
\end{array}\right]\left[\begin{array}{l}
b \\
\bar{c} \\
b
\end{array}\right] \bar{q} S} \\
{\left[\begin{array}{c}
X \\
Y \\
Z
\end{array}\right]=m\left[\begin{array}{l}
a_{x} \\
a_{y} \\
a_{z}
\end{array}\right]} \\
{\left[\begin{array}{c}
L \\
M \\
N
\end{array}\right]=\left[\begin{array}{ccc}
I_{x x} & -I_{x y} & -I_{x z} \\
-I_{y x} & I_{y y} & -I_{y z} \\
I_{z x} & -I_{z y} & I_{z z}
\end{array}\right] \dot{\omega}}
\end{gathered}
$$

Here, $a$ denotes acceleration along each body axis as indicated by the subscript, $I$ denotes the various moments of inertia and $\dot{\omega}$ represents the angular acceleration vector containing the $x, y$ and $z$ axes. The flight simulator runs in real time calculating at $100 \mathrm{~Hz}$. Flight visualisation is done through FlightGear.

\section{A. Control Surface Rate Limitations}

Control surface rate limitations must be considered in the modelling environment to produce feasible results. A DO can, given ideal sensors, estimate disturbances very rapidly. However, the ability of the system to act on these estimates is limited by the bandwidth of the control outputs. To match the response rate of the controls available on the physical

\footnotetext{
${ }^{2}$ http://www.mathworks.com/tagteam/9477_aero.pdf
} 
$\mathrm{X} 8$ being simulated, the rates given in (10) were imposed in the simulation. Here, $\Delta \epsilon$ represents the rate of elevator deflection and $\Delta T$ represents the rate of engine thrust output. In terms of time, this means the aircraft elevator surfaces can go from minimum to maximum deflection in $0.25 \mathrm{~s}$ and similarly thrust can go from minimum to maximum in $0.5 \mathrm{~s}$. This is representative of the control rates available on the physical aircraft.

$$
\begin{aligned}
|\Delta \epsilon| & \leq 4 \mathrm{rad} / \mathrm{s} \\
|\Delta T| & \leq 200 \% / \mathrm{s}
\end{aligned}
$$

\section{B. Dryden Wind Model}

An accurate gust model is essential for this simulation study. The Dryden model is commonly used in literature [12] [13] and furthermore is approved by US Military Specification [14] as an appropriate means for simulating gusts. The Dryden model passes band limited white noise through forming filters to produce a stochastic gust output based on the given airspeed and height of the aircraft. The resulting output are gusts which sufficiently represent the random gusts which would be encountered in flight. A more rigorous mathematical definition can be found in literature [15].

This model is readily available in Simulink as part of the Aerospace block set, and was chosen as the model for gust simulation in this study.

\section{Control Design}

\section{A. Feedback Control Design}

The objective of the designed feedback controller is to regulate the aircraft states $(u, w, q, \theta$ and $h)$ as defined in Section II at their trim condition whilst also allowing $u$ and $h$ reference tracking. This allows for an initial demonstration of the gust alleviation performance in path following as well as attitude control. A Linear Quadratic Regulator (LQR) was chosen as the control scheme as it is well suited to the task of maintaining a trim condition and furthermore it is commonly formulated around state-space models, which is the same model proposed for the DO in later sections. An LQR offers good regulation around a trim condition however, some tracking performance is also required in the form of height reference tracking. For this reason, the LQR is augmented with integral action, to become an Linear Quadratic Regulator with Integral action (LQI).

Linear Quadratic design theory focuses around minimising a cost function defined in (11) where $x$ and $u$ are the same state and control variables as defined in the state-space model. $Q$ and $R$ are cost matrices which can be used to define the weighting on the change in each state $(Q)$ and the control action applied for each output $(R)$.

$$
J=\frac{1}{2} \int_{\infty}^{0}\left(x^{T} Q x+u^{T} R u\right) d t
$$

Integral augmentation is achieved by modifying $x$ to be a matrix of two sets of states, as defined in (12) where the new state, $e$, is the integral of the error between $y$, the system output and $r$, the reference signal. Substituting $x_{e}$ for $x$ in (11) now includes integral action.

$$
x_{e}=\left[\begin{array}{c}
\dot{x} \\
\dot{e}
\end{array}\right]=\left[\begin{array}{c}
A x+B u \\
y-r
\end{array}\right]=\left[\begin{array}{c}
A x+B u \\
C x-r
\end{array}\right]
$$

More detailed examples of LQ design theory can be found in literature [16]. For this work, the state-space model is already available. The performance of the feedback controller was not subject to significant tuning as it is the difference provided by the addition of the DO compensation signal while using the same baseline feedback controller which is of interest. As such, the $Q$ and $R$ matrices were chosen simply in an identity form and scaled to avoid control saturation.

\section{B. Disturbance Observer Definition}

The DO is added into the state-space model by adding disturbance and uncertainty terms which represent effects that cannot be captured by the linear model in (2). In this system these effects are the modelling errors of the nonlinear aircraft dynamics and the external disturbances. This is shown in (13). Here, $B_{d}$ is the disturbance matrix and $d_{x}$ are the disturbances on the states. $B_{d}$ in this case takes the form of a $I_{5 x 5}$ matrix. The difference in form of $B$ and $B_{d}$ represents a mismatched solution; that is to say a solution where a direct control surface is not available to act on each disturbance. This situation requires careful design of the feedforward control strategy.

$$
\dot{x}=A x+B u+B_{d} d_{x}
$$

Another key feature of DOBC is the ability to account for modelling uncertainties. This is done by treating such uncertainties as another disturbance on the system, an internal disturbance. Combining these internal disturbances with the external disturbances as given in (13) they are defined together as lumped disturbances. This is given in (14)

$$
d_{l x}=B_{d} d_{x}+\left(A_{t}-A\right) x+\left(B_{t}-B\right) u
$$

Here, $d_{l x}$ represents the now lumped disturbances, $A_{t}$ and $B_{t}$ are the true matrices which would ideally describe the system being modelled; $A$ and $B$ are the matrices which were developed in Section II to represent the true matrices. It is expected that these will differ from the true values. This formulation of the observer shows that, in the case where the nominal matrices match the actual system matrices (i.e. a perfect model) the lumped disturbances consist purely of external disturbances. Conversely, in the absence of external disturbances the lumped disturbances only represents the internal disturbances due to modelling uncertainties. In reality however, both internal and external disturbances will be present. This property is useful in this case as the DO is designed using the linearised state-space model, but will be applied to a fully non-linear simulation of the aircraft. Eq. (14) indicates that the resulting modelling inaccuracies can be accounted for while still estimating the targeted external disturbance. Another less obvious benefit can be found when considering the target application of this technique; 
small, low cost UASs. These UASs are often hand built and therefore experience variation in their properties between aircraft. Using this technique, it should be possible to design a DO based on a single model of the aircraft and allow the DO to account for the varying modelling inaccuracies which are expected. This significantly improves the real world applicability of the technique.

\section{Feedforward Control Design}

To estimate the lumped disturbances, a disturbance observer is required. For this work, a linear state-space observer was employed. This is a widely used and understood modelling method which is also easy to implement; the limitation being the linear nature of the observer. This work hopes to demonstrate the feasibility of using such a linear observer to improve the performance of a fully non-linear aircraft. The observer is given in (15) where $\hat{d}_{l x}$ is the estimate of the lumped disturbances, $z$ is an auxiliary internal state and $L$ is a gain matrix which is to be designed [17].

$$
\left\{\begin{array}{l}
\dot{z}=-L(z+L x)-L\left(A_{n} x+B_{n} u\right) \\
\hat{d}_{l x}=z+L x
\end{array}\right.
$$

Where $\mathrm{L}$ is conventionally chosen as an identity matrix with the magnitude of each element dictating the response rate of the observer to the related parameter. Generally speaking, a higher $L$ will result in a more sensitive observer with a faster response rate. The trade off is an increased sensitivity to sensor noise, hence for physical application this will need to be tuned for best performance.

The disturbance estimate $\hat{d_{l x}}$ from the observer can asymptotically track the lumped disturbances $d_{l x}$ if the observer gain matrix $L$ is chosen such that $-L B_{d}$ satisfies the Hurwitz stability criteria. This can be shown by studying the estimation error dynamics with an assumption that the lumped disturbances vary slowly and tend to a constant value. This represents the case for steady disturbances, which are examined in the initial part of the simulation section.

$$
\begin{aligned}
\dot{e}_{d}= & \dot{\hat{d}}_{l} x-\dot{d}_{l} x \\
= & \dot{z}+L \dot{x}-\dot{d}_{l} x \\
= & -L B_{d} \hat{d}_{l x}-L\left(A_{n} x+B_{n} u\right)+ \\
& L\left(A_{n} x+B_{n} u+B_{d} d_{l x}\right)-\dot{d}_{l x} \\
= & -L B_{d} e_{d}-\dot{d}_{l x}
\end{aligned}
$$

It can be seen from (16) that the error is stable if $-L B_{d}$ is Hurwitz and the disturbance $d_{l x}$ is bounded and satisfies the condition $\lim _{t \rightarrow \infty} \dot{d}_{l x}=0$. This assumption is based on the knowledge that the observer dynamics vary at a higher rate than that of the disturbance itself. At this point, an observer has been designed which is able to provide estimates of the disturbances to the system. It remains to design a control law which is able to use these estimates to reject their influence from the system.

Using the LQI designed in Section IV-A and solving (17) yields a feedforward gain matrix to be applied to the disturbance estimates [18].

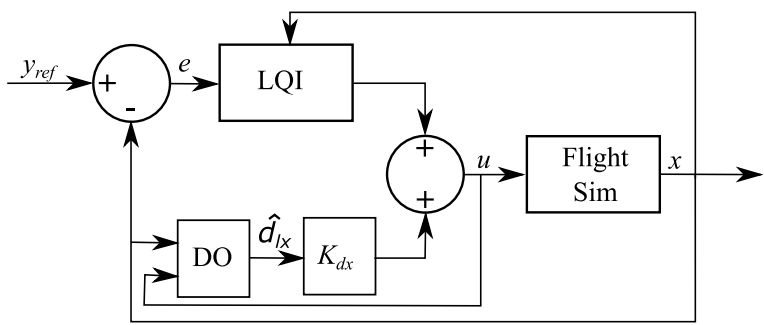

Fig. 3: An overview of the control structure for the baseline LQI controller and the compensation signal from the DO.

$$
\begin{aligned}
K_{d x} & =\left[D_{n}-\left(C_{n}+D_{n} K_{L Q I}\right)\left(A_{n}+B_{n} K_{L Q I}\right)^{-1} B_{n}\right]^{-1} \\
& \times\left(C_{n}+D_{n} K_{L Q I}\right) \times\left(A_{n}+B_{n} K_{L Q I}\right)^{-1} B_{l d}
\end{aligned}
$$

The LQI control law is shown in (18). To expand $u$ to include the feedforward compensation signal is a simple addition process and the overall control law with DO compensation signal is given in (19).

$$
\begin{gathered}
u=K_{L Q I} x \\
u=K_{L Q I} x+K_{d x} \hat{d}_{l x}
\end{gathered}
$$

At this point the complete DO controller has been designed. An overview of the control structure is given in Fig 3.

\section{Simulation}

For all the results presented in this section, the flight simulation environment described in Section III was used. This includes the fully non-linear Simulink model of the X8 with a $0.01 \mathrm{~s}$ time step to ensure proper capture of rapid dynamics. The same linear DO was employed throughout with no changes to either the feedback or feedforward gains for the various disturbances. The trimmed aircraft state is given in (20) where ${ }^{*}$ denotes the trim state of the associated variable.

$$
\begin{array}{cc}
u^{*} & 15.0 \mathrm{~m} / \mathrm{s} \\
w^{*} & -0.05 \mathrm{~m} / \mathrm{s} \\
q^{*}= & 0^{\circ} / \mathrm{s} \\
\theta^{*} & -0.2^{\circ} \\
h^{*} & 300 \mathrm{~m}
\end{array}
$$

\section{A. Reference Tracking Performance}

An ideal DO would not augment the control output due to a change in reference in the absence of uncertainties. In reality, internal disturbances (modelling errors) are likely to result in an augmented response. To study this, the first simulation conducted was a $30 \mathrm{~m}$ change in target height. The step input was passed through a second order filter to smooth the reference signal. The resulting response is shown in Fig 4.

In this case, the proposed control scheme tracks the reference height more accurately than the LQI alone, as shown 

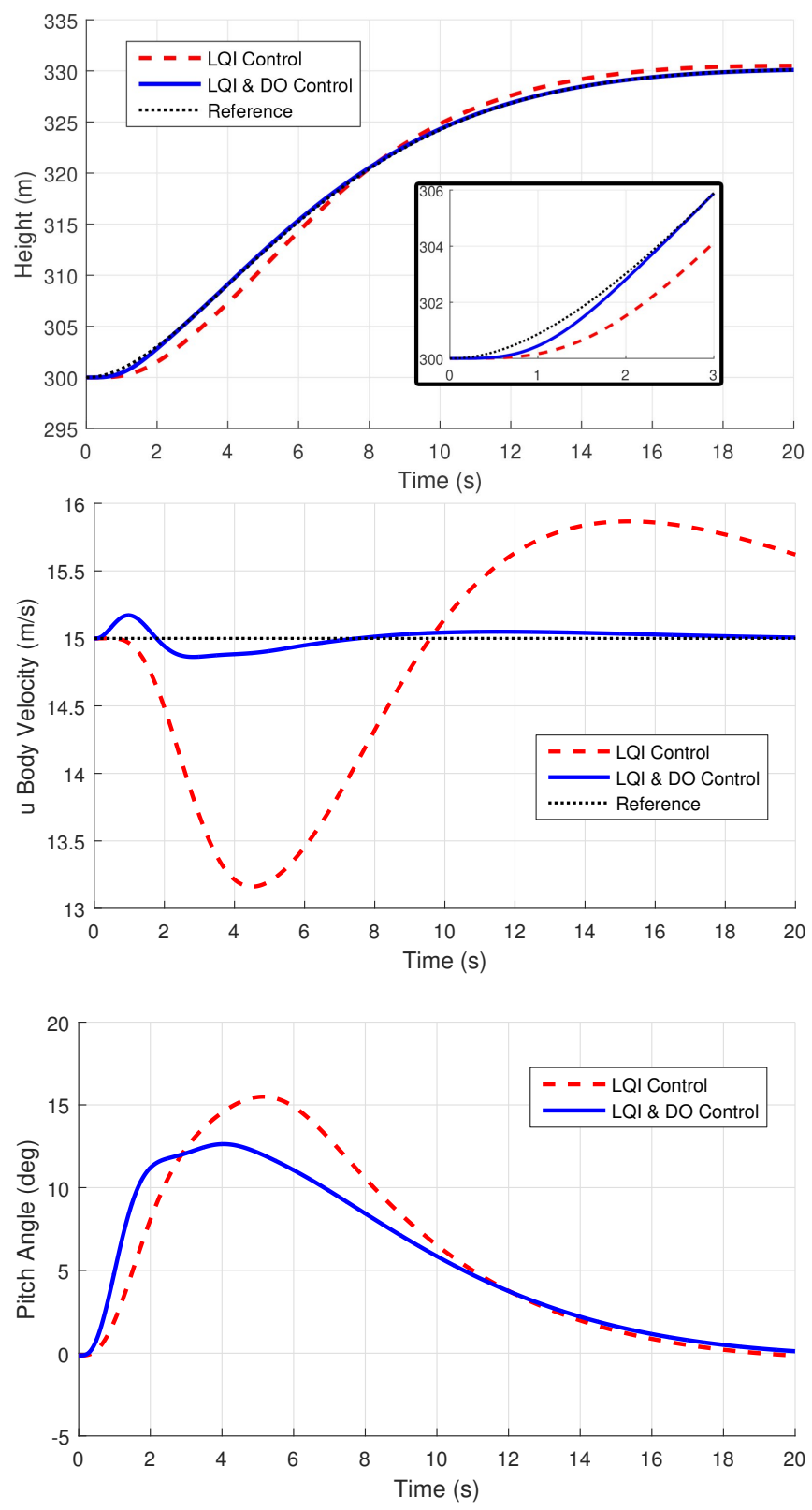

Fig. 4: A comparison of aircraft states during the height step change. Figure (a) includes an enlargement of the initial period.

in Fig 4. This is because the DO perceives the disturbance on forward velocity introduced by pitching up more quickly than the LQI only control, and as can be seen in Fig5, the throttle setting is increased well ahead of the LQI controller. This results in a much better tracking of the reference forward speed. This also means that overshoot of the height reference $(t=8 s)$ is removed as the integral component of the controller does not accrue any significant value.

\section{B. Horizontal Gust Disturbance Rejection}

The focus of the addition of a DOBC is to improve control performance of small UASs in the presence of external
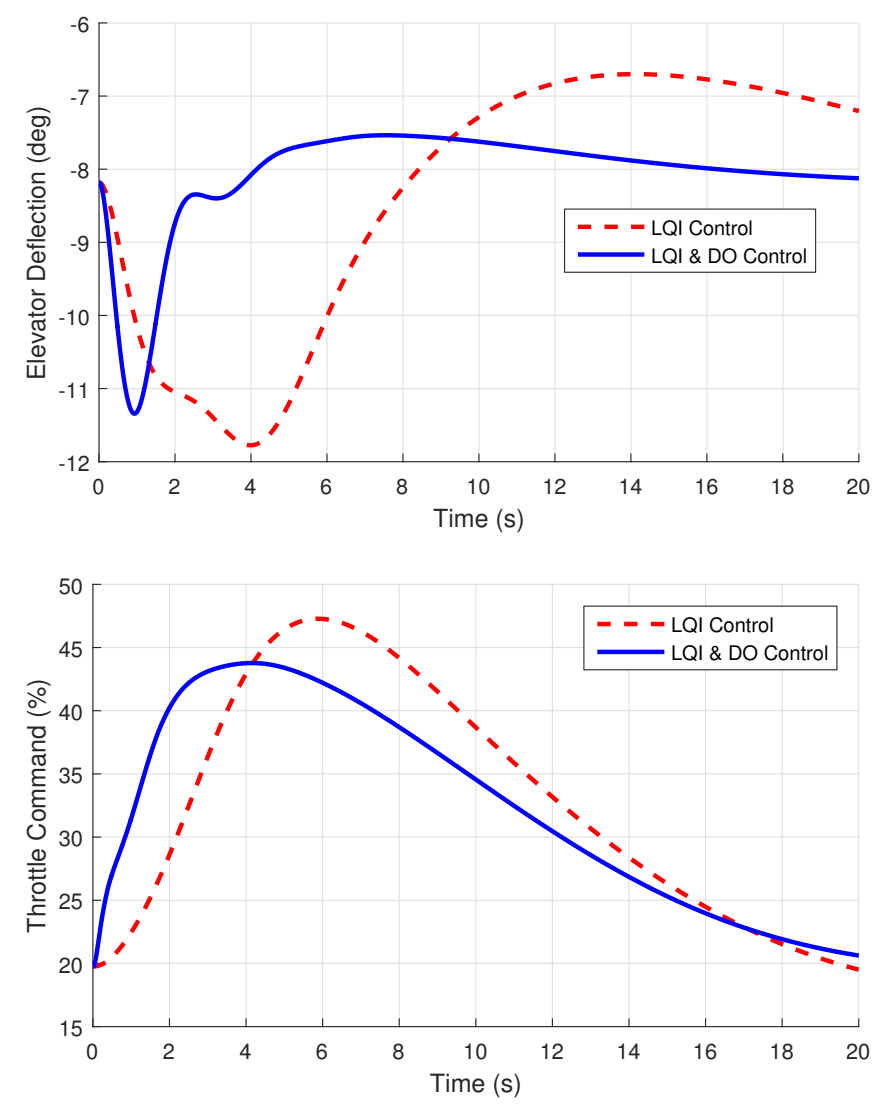

Fig. 5: A comparison of control surface deflections during the height step change.

disturbances. For this work, wind gusts are the external disturbance which are considered. This can be further broken down into horizontal $\left(w_{x}\right)$ and vertical $\left(w_{h}\right)$ gusts as defined in Fig 1. These disturbances will affect the aircraft dynamics in different ways. In this work, horizontal position is not considered and as such horizontal gust disturbances will mainly affect the aircraft attitude. As attitude is directly affected by control outputs it is expected the aircraft will recover quickly. In the vertical channel, the proposed controller is also controlling position in terms of height. It is expected that the recovery of height will be slower than that of attitude as not only will the initial disturbance need to be alleviated but position will also need to be recovered.

The first considered disturbance is sudden addition of a gust with magnitude $w_{x}=-5 \mathrm{~m} / \mathrm{s}$. This represents a significant disturbance when compared to the airspeed of $15 \mathrm{~m} / \mathrm{s}$. This disturbance is passed through a rate limiter which is limits the rate to $\pm 20 \mathrm{~m} / \mathrm{s}^{2}$, representing a very sharp gust. The resulting response is shown in Fig 6. The addition of the compensation signal shows a significant reduction in the height, forward speed and pitch perturbation experienced by the aircraft. The effect on control output is clearly visible in Fig 7; throttle is immediately increased significantly over the LQI controller, which means that the elevator deflection required is also greatly reduced. the magnitude of initial 

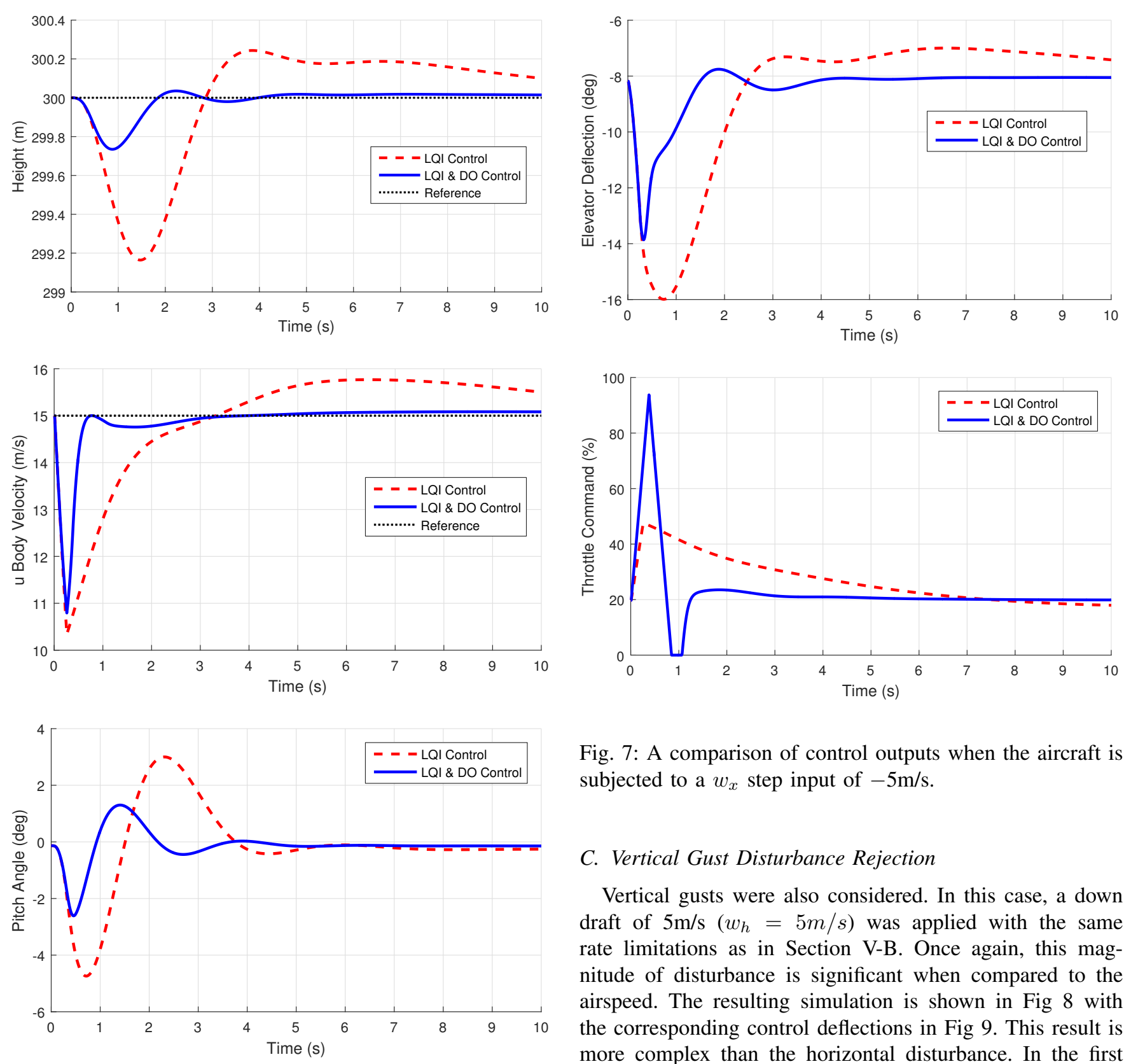

Fig. 6: A comparison of aircraft states when subjected to a $w_{x}$ step input of $-5 \mathrm{~m} / \mathrm{s}$.

$u$ velocity perturbation is not decreased by a significant amount, although the reference speed is recovered roughly 3 times faster than LQI only control. As a result, the height disturbance is reduced by around $60 \%$ and the reference is recovered more quickly. Furthermore, the oscillations experienced in pitch angle are reduced in magnitude. Overall, the addition of the compensation signal has resulted in a significantly more stable platform which retains it's reference condition far better than LQI control alone.

Fig. 7: A comparison of control outputs when the aircraft is subjected to a $w_{x}$ step input of $-5 \mathrm{~m} / \mathrm{s}$.

\section{Vertical Gust Disturbance Rejection}

Vertical gusts were also considered. In this case, a down draft of $5 \mathrm{~m} / \mathrm{s}\left(w_{h}=5 \mathrm{~m} / \mathrm{s}\right)$ was applied with the same rate limitations as in Section V-B. Once again, this magnitude of disturbance is significant when compared to the airspeed. The resulting simulation is shown in Fig 8 with the corresponding control deflections in Fig 9. This result is more complex than the horizontal disturbance. In the first second post disturbance, the height tracking performance is not improved over LQI control. This can be explained by studying the forward speed result, which does show a difference when compared to LQI only control. The forward speed is maintained much more closely to the reference command with the compensation signal active; the maximum deviation with compensation signal active was only $26 \%$ the magnitude of LQI control alone. This subsequently allows for recovery of the lost height much more quickly. The total height loss with the compensation signal is reduced by $57 \%$ and more significantly, the height is returned to within $0.5 \mathrm{~m}$ of the reference height $1.5 \mathrm{~s}$ post disturbance with the compensation signal active as opposed to $7.3 \mathrm{~s}$ without. This represents a significant improvement in performance. It can be seen from the pitch data in Fig 8, along with the control input data in Fig 9 that with the compensation signal active the aircraft settles to the appropriate trim state 

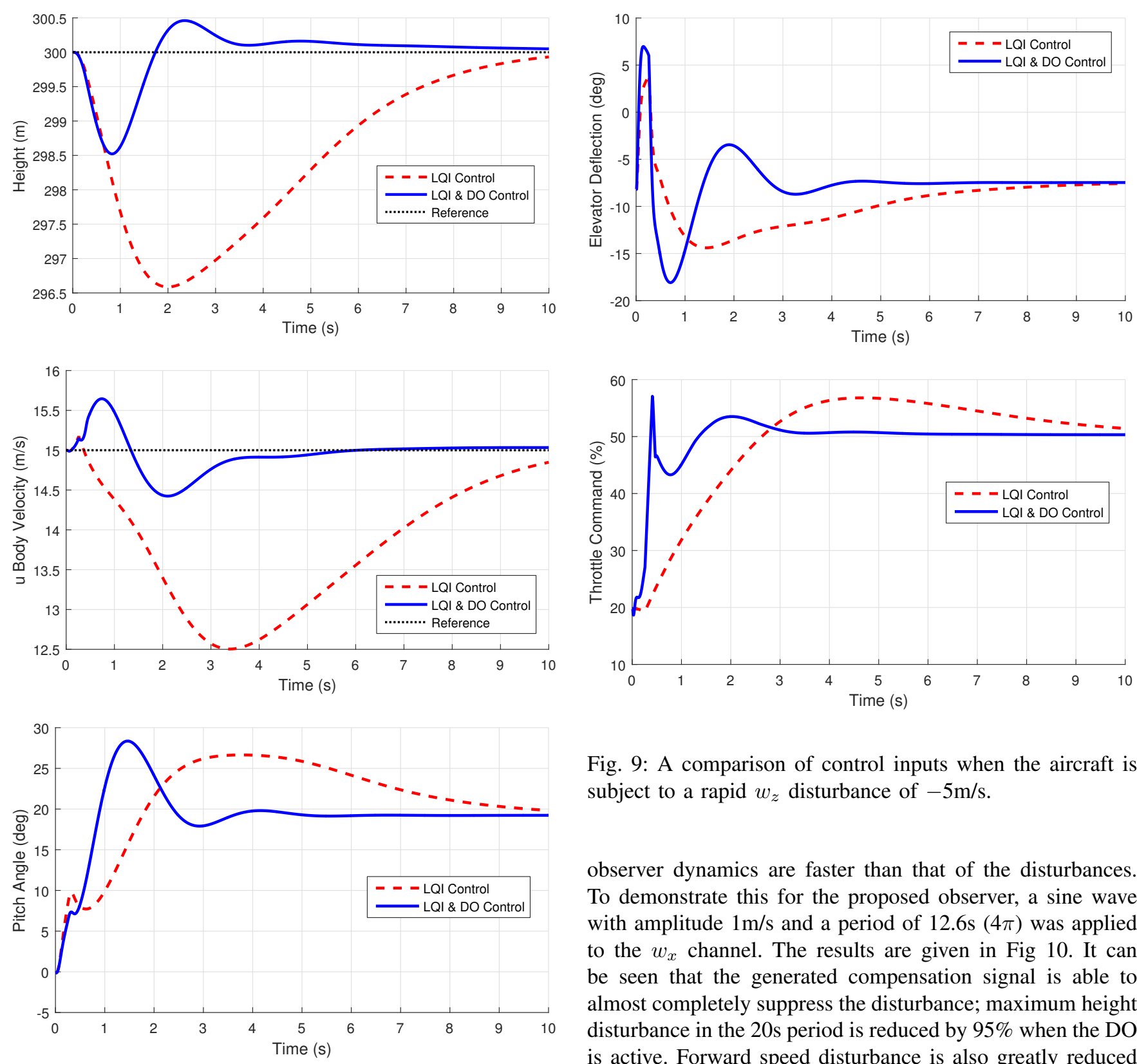

Fig. 8: A comparison of aircraft states when subject to a rapid $w_{z}$ disturbance of $-5 \mathrm{~m} / \mathrm{s}$.

of $\theta=19.2^{\circ}, \delta T=50.3 \%, \delta \epsilon=-7.4^{\circ}$ which maintains the reference commands in the presence of the gust; the LQI only controller is approaching this condition by the end of the simulation. This demonstrates the operating principle of Disturbance Observer Based Control (DOBC) very well; the disturbance is estimated and the appropriate control signal generated rapidly.

\section{Sine Wave Disturbance}

Eq. 16 demonstrated DO stability with the assumption that $\lim _{t \rightarrow \infty} \dot{d}=0$. However, as was shown in [19], the disturbance estimates can track the disturbances if the

Fig. 9: A comparison of control inputs when the aircraft is subject to a rapid $w_{z}$ disturbance of $-5 \mathrm{~m} / \mathrm{s}$.

observer dynamics are faster than that of the disturbances. To demonstrate this for the proposed observer, a sine wave with amplitude $1 \mathrm{~m} / \mathrm{s}$ and a period of $12.6 \mathrm{~s}(4 \pi)$ was applied to the $w_{x}$ channel. The results are given in Fig 10. It can be seen that the generated compensation signal is able to almost completely suppress the disturbance; maximum height disturbance in the 20 s period is reduced by $95 \%$ when the DO is active. Forward speed disturbance is also greatly reduced by $96 \%$, representing a much more stable aircraft. Similar improvements can be seen in the pitch angle disturbance rejection. This very successful disturbance rejection is made possible by the bandwidth of the disturbance being within that of both available control surfaces, meaning that the required compensation signal is always realisable by the aircraft. However, this regular pattern is not representative of real world conditions; for this, the Dryden wind model is required.

\section{E. Dryden Wind Disturbances}

For the final run of simulations the Dryden wind model, as discussed in Section III-B, was used to generate stochastic gusting wind which was applied to the $w_{x}$ channel. To further increase the workload, an initial sharp gust of $w_{x}=-5 \mathrm{~m} / \mathrm{s}$ was applied at the same time with the same characteristics as in Section V-B. The results are shown in Fig 11. The 

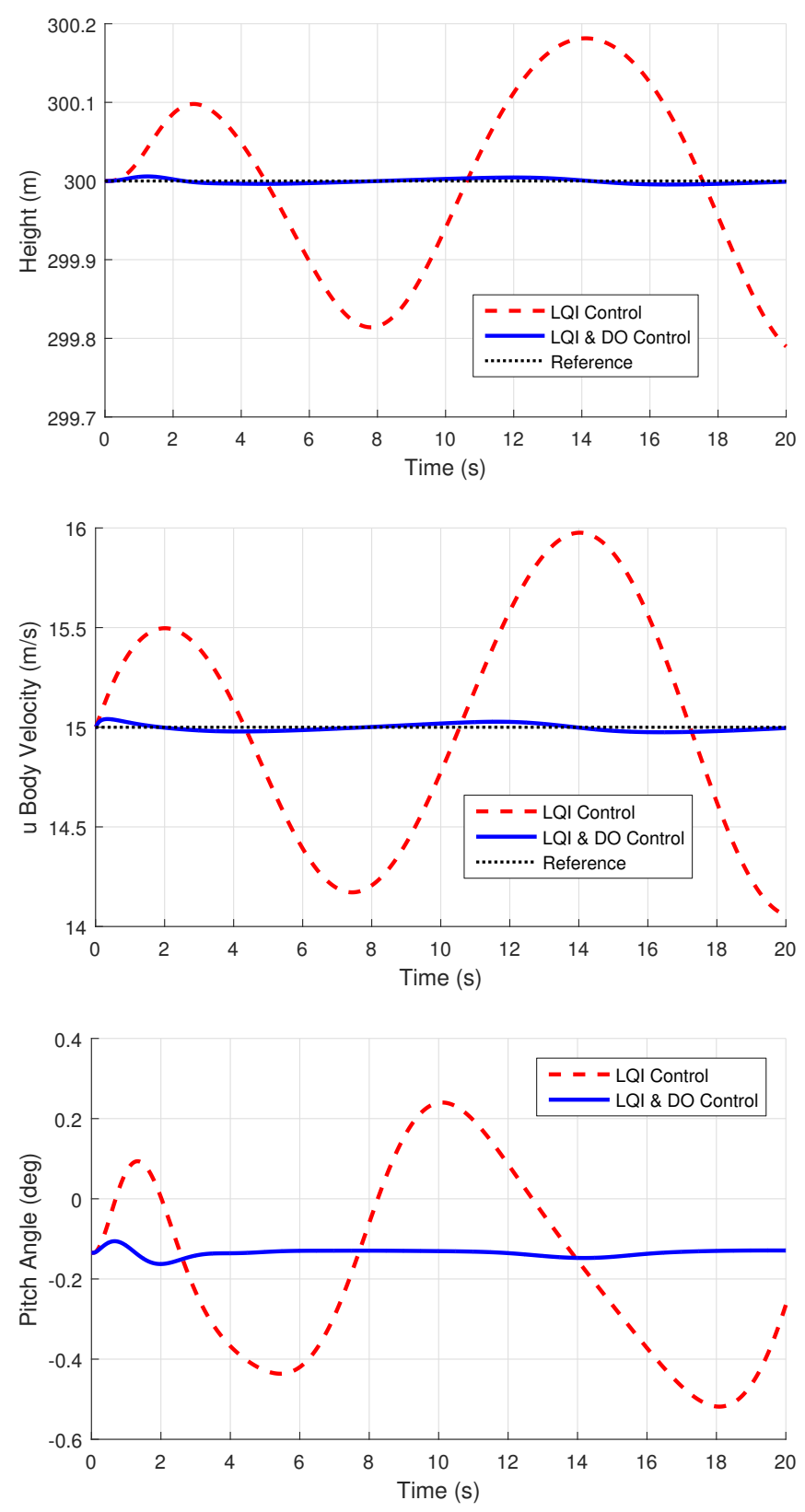

Fig. 10: A comparison of aircraft states when subjected to a sine wave disturbance

initial disturbance rejection on the height channel shows the same performance as discussed in Section V-B as expected. However, the subsequent performance for height holding is greatly improved, especially when considering the portions of graph where the height is pushed below the reference trajectory by the gusts. This is due to the compensation signal being able to demand additional acceleration with throttle. The acceleration generated by additional throttle is significantly greater than the deceleration resulting from reducing throttle (i.e. drag), meaning the control system can accelerate the aircraft much more quickly than it can decelerate it; herein lies the reason for improved disturbance rejection when acceleration is required rather than deceleration. Similar behaviour can be seen in the $u$ velocity graph. Unfortunately this graph becomes difficult to read clearly due to the noisy nature of Dryden gusts. However, it can be still be seen that the addition of the DO has reduced the perturbations here when compared to the LQI only control. This is especially noticeable for the periods where $u$ velocity falls below $15 \mathrm{~m} / \mathrm{s}$, which is an important result; the DO is able to keep the aircraft well clear of its' stall condition, which occurs at around $10 \mathrm{~m} / \mathrm{s}$. The LQI only controller breaches this briefly in the initial period and approaches it again between $t=14 \mathrm{~s}$ and $t=20 \mathrm{~s}$. The associated height holding performance is also greatly improved for this period. Keeping the aircraft away from the stall condition means that proper control authority is retained for the elevators, which rely on dynamic pressure. Furthermore, a stall itself is a dangerous flight condition and keeping the aircraft clear of this is extremely beneficial. Considering a landing scenario, if such a stall occurred close to the ground the aircraft would be in great danger, especially when coupled with the associated height loss. This shows promise for the intended future application of this technique to a landing controller.

The addition of the DO has improved performance even in the presence of Dryden winds. An initial concern of the work was that a linear observer would not be able to produce accurate or reasonable compensation signals when the aircraft departs significantly from the trim condition about which the linearised model was created. It is interesting to note from the results that the addition of the DO results in the aircraft remaining closer to the trim condition, which allows the linear model to remain reasonably accurate.

\section{CONClusions And Future Work}

The method outlined in this work has shown that a linear disturbance observer can be used to alleviate the influence of a range of gust conditions on the longitudinal dynamics of a small UAS. For step inputs, the rapid estimation of disturbances allows the DO to generate a compensation signal which is able to return the UAS to the nominal condition very quickly. This was achieved with less perturbation in both the height and forward speed, with the DO enabled controller generally resulting in flight much closer to the reference command. It has also been demonstrated that for continuously varying disturbances which vary at a rate within both of the control surfaces' bandwidths, the DO was able to alleviate the effect of the gusts extremely well. The final simulation made use of Dryden gusts as a source of unpredictable, realistic disturbances. In these simulations the DO was able to keep the aircraft much closer to the reference conditions than LQI only control. Furthermore, in this section the LQI controller approached the stall condition of the aircraft while the compensation signal was able to keep the aircraft well away from this situation, and with minimal height loss. This is an important result for the future work which aims at producing a landing controller able to safely land small UASs in the presence of gusts. These initial result show that a DO certainly is capable of greatly improving the 

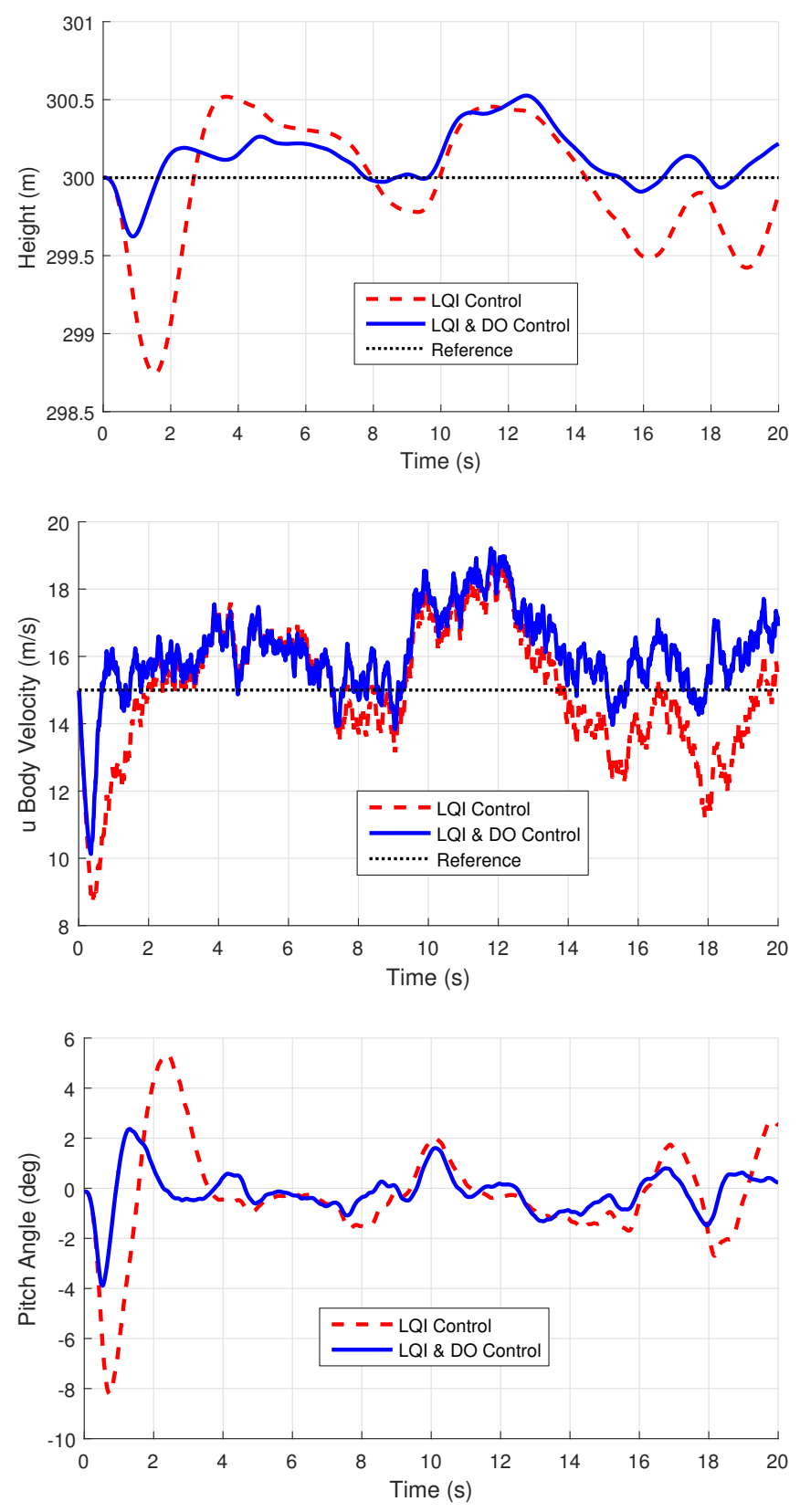

Fig. 11: A comparison of aircraft states when subjected to a constant $w_{x}=-5 \mathrm{~m} / \mathrm{s}$ disturbance and simultaneous Dryden gusts in the $w_{x}$ channel

performance in such conditions. An unexpected outcome of the work was the result that, even when subject to Dryden gusts, the linear DO keeps the aircraft very close to the linearisation point. This aids it in performance as it can more accurately estimate distrubances in the region where the linear model is most accurate.

Future work has aims to produce a landing control for small UASs which uses a disturbance observer to improve performance and safety in the presence of wind. This work has demonstrated the feasibility of a linear DO for such tasks, so a non-linear observer is not an immediate focus; two short term goals are planned. Firstly, a study into using a DO to mitigate the problem of control saturation. Secondly, a lateral channel DO is also required to be able to address the landing problem appropriately.

\section{REFERENCES}

[1] M. Abdulrahim, S. Watkins, R. Segal, M. Marino, and J. Sheridan, "Dynamic sensitivity to atmospheric turbulence of unmanned air vehicles with varying configuration," Journal of aircraft, vol. 47, no. 6, pp. 1873-1883, 2010.

[2] S. Watkins, J. Milbank, B. J. Loxton, and W. H. Melbourne, "Atmospheric winds and their implications for microair vehicles," AIAA Journal, vol. 44, no. 11, pp. 2591-2600, Nov. 2006. [Online]. Available: http://arc.aiaa.org/doi/abs/10.2514/1.22670

[3] A. Mohamed, S. Watkins, R. Clothier, M. Abdulrahim, K. Massey, and R. Sabatini, "Fixed-wing mav attitude stability in atmospheric turbulence, part 2: Investigating biologically-inspired sensors," Progress in Aerospace Sciences, vol. 71, pp. 1-13, 2014.

[4] A. Mohamed, R. Clothier, S. Watkins, R. Sabatini, and M. Abdulrahim, "Fixed-wing mav attitude stability in atmospheric turbulence, part 1: Suitability of conventional sensors," Progress in Aerospace Sciences, vol. 70, pp. 69-82, 2014.

[5] M. Coombes, W. Eaton, O. McAree, and W.-H. Chen, "Development of a generic network enabled autonomous vehicle system," in Control (CONTROL), 2014 UKACC International Conference on, July 2014, pp. 621-627.

[6] R. W. Beard and T. W. McLain, Small unmanned aircraft: Theory and practice. Princeton University Press, 2012.

[7] T. A. Johansen, A. Cristafaro, K. Sørensen, J. M. Hansen, and T. I. Fossen, "On estimation of wind velocity, angle-of-attack and sideslip angle of small uavs using standard sensors," in International Conference on Unmanned Aircraft Systems, 2015.

[8] A. A. Rodriguez, O. Cifdaloz, M. Phielipp, and J. Dickeson, "Description of a modeling, simulation, animation, and real-time control (mosart) environment for a class of 6-dof dynamical systems," in American Control Conference, 2007. ACC'07. IEEE, 2007, pp. 25152520.

[9] P. Lu and Q. Geng, "Real-time simulation system for uav based on matlab/simulink," in Computing, Control and Industrial Engineering (CCIE), 2011 IEEE 2nd International Conference on, vol. 1. IEEE, 2011, pp. 399-404.

[10] M. D. Ilg, "Guidance, navigation, and control for munitions a thesis submitted to the faculty of," Ph.D. dissertation, Drexel University, 2008.

[11] J. E. Steck, K. Rokhsaz, U. Pesonen, S. Bruner, and N. Duerksen, "Simulation and flight test assessment of safety benefits and certification aspects of advanced flight control systems," Federal Aviation Administration, Final Rept. DOT/FAA/AR-03/51, 2003.

[12] J. How, E. King, and Y. Kuwata, "Flight demonstrations of cooperative control for uav teams," in AIAA 3rd unmanned unlimited technical conference, workshop and exhibit, 2004, pp. 20-23.

[13] A. Dogan, T. A. Lewis, and W. Blake, "Flight data analysis and simulation of wind effects during aerial refueling," Journal of Aircraft, vol. 45, no. 6, pp. 2036-2048, 2008.

[14] "Flying qualities of piloted aircraft," United States Department of Defense, Tech. Rep., 1997.

[15] J. W. Langelaan, N. Alley, and J. Neidhoefer, "Wind field estimation for small unmanned aerial vehicles," Journal of Guidance, Control, and Dynamics, vol. 34, no. 4, pp. 1016-1030, 2011.

[16] K. P. Groves, D. O. Sigthorsson, A. Serrani, S. Yurkovich, M. A Bolender, and D. B. Doman, Reference command tracking for a linearized model of an air-breathing hypersonic vehicle. Defense Technical Information Center, 2005.

[17] S. Li, J. Yang, W.-H. Chen, and X. Chen, Disturbance Observer-Based Control: Methods and Applications. CRC Press, 2014.

[18] J. Yang, A. Zolotas, W.-H. Chen, K. Michail, and S. Li, "Robust control of nonlinear maglev suspension system with mismatched uncertainties via dobc approach," ISA transactions, vol. 50, no. 3, pp. 389-396, 2011.

[19] W.-H. Chen, D. J. Ballance, P. J. Gawthrop, and J. O'Reilly, “A nonlinear disturbance observer for robotic manipulators," Industrial Electronics, IEEE Transactions on, vol. 47, no. 4, pp. 932-938, 2000. 